\title{
Digital electron microscopic examination of human sural nerve biopsies
}

\author{
K. A. Sullivan, ${ }^{1}$ M. S. Brown, ${ }^{2}$ L. Harmon, ${ }^{3}$ and D. A. Greene ${ }^{4}$ \\ Department of Neurology, ${ }^{1}$ Department of Biostatistics, University of Michigan, ${ }^{2}$ Ann Arbor, Michigan, Nonlinear Dynamics ${ }^{3}$ \\ Ann Arbor, Michigan, and Sanofi-Synthelab, New York, New York ${ }^{4}$
}

\begin{abstract}
Diabetic peripheral polyneuropathy is characterized by axonal degeneration and regeneration as well as by Schwann cell and microvascular changes. These changes have been described at both the light (LM) and the electron microscopic (EM) levels; however, EM has not been applied to large clinical trials. Our goal was to adapt the rigorous techniques used for quantifying human biopsies with LM image analysis to accommodate ultrastructural analyses. We applied digital image capture and analysis to the ultrastructural examination of axons in sural nerve biopsies from diabetic patients enrolled in a multicenter clinical trial. The selection of sural nerve biopsies was based on the quality of specimen fixation, absence of physical distortion, and nerve fascicle size $\left(\geq 100000 ; \leq 425000 \mu \mathrm{m}^{2}\right)$. Thin sections were collected on formvar-coated slot grids, stabilized with carbon and scanned on a Phillips CM100 transmission electron microscope. Digital images were captured with a Kodak Megaplus 1.6 camera. A montage was constructed using software derived from aerial mapping applications, and this virtual image was viewed by EM readers. Computer-assisted analyses included identification and labeling of individual axons and axons within regenerating clusters. The average density of regenerating myelinated axon clusters per $\mathrm{mm}^{2}$ was $65.8 \pm 5.1$, range of $0-412(n=193)$. These techniques increase the number of samples that may be analyzed by EM and extend the use of this technique to clinical trials using tissue biopsies as a primary endpoint.
\end{abstract}

Key words: diabetes, digital imaging, electron microscopy, human sural nerve, neuropathy

\section{Introduction}

Diabetes mellitus affects over 16 million people in the US, and the number of people with diabetes increases by $5 \%$ per year (Herman et al., 1984). Up to half of these patients develop diabetic polyneuropathy (DPN) over the course of their disease (Greene et al., 1997). DPN is characterized by degeneration and attempted regeneration of myelinated and unmye-

Address correspondence to: Dr. K. A. Sullivan, Department of Neurology, 1331 East Ann St. Building, Room 6131, Ann Arbor, MI 48109-0580, USA. Tel: +1-734-615-6231; Fax: +1-734-647-2307; E-mail: ksulliva@umich.edu linated axons (Black and Lasek, 1980; Said et al., 1983; Dyck et al., 1986; Llewelyn et al., 1988; 1991; Said, 1996). Over time, regeneration fails to keep pace with the degenerative process, resulting in a decrease in the number of myelinated nerve fibers per $\mathrm{mm}^{2}$ (myelinated nerve fiber density, MNFD), distal loss of sensation, and muscle weakness (Greene et al., 1997).

Patient treatment is aimed at preventing fiber loss, with consequent amelioration of signs and symptoms. Measurements to assess treatment effectiveness include nerve conduction testing (Stevens et al., 1993b; Comi et al., 1999), sensory testing (Stevens et al., 1993b), and nerve morphology (Feldman et al., 
1994a; 1994b; Greene et al., 1997). Morphological parameters examined in sural nerve biopsies include MNFD, myelinated fiber size frequency histograms, and teased fiber analysis. These methods monitor the myelinated fiber populations affected by diabetes (Feldman et al., 1994a; 1994b; Greene et al., 1997) and help define the degree of damage. Previous light microscopy studies in both human diabetic patients and animal models of diabetic neuropathy report modest increases in MNFD in response to treatment (Sima et al., 1988; 1993b); however, it is not clear from these investigations as to how MNFD is maintained. Treatment may increase MNFD over time by decreasing the number of degenerating axons, increasing the number of regenerating axons, or a combination of the two.

Regenerating myelinated nerve fibers are defined as multiple, myelinated fibers surrounded by a common basal lamina (Sima et al., 1988; Britland et al., 1990; Greene et al., 1997). This definition derives from the observations of degeneration and regeneration in the peripheral nervous system (Greene et al., 1997; Sima et al., 1988; Britland et al., 1990). The original healthy myelinated fiber is surrounded by myelinating Schwann cells (SCs) that create and support an encircling basal lamina. Following axonal damage, the SCs de-differentiate and undergo mitosis to form a Büngner Band comprised of dividing SCs within the original basal laminar tube. Contact by growing axonal projections induces the surrounding SCs to differentiate and begin myelination. This process results in multiple axon sprouts in varying states of regrowth and myelination residing within the original basal laminar tube. As the new fibers mature, the original basal lamina is lost, leaving a group of small myelinated fibers, each with its own basal lamina, in the place of the original nerve fiber.

Because the thickness of the basal lamina is below the resolution of light microscopy (LM), electron microscopy (EM) is necessary to fully examine the morphological characteristics of regenerating axons and accurately determine their number and relation to the basal lamina. LM analysis of regenerating axons cannot distinguish between true regeneration contained within a continuous basal lamina and small randomly grouped myelinated fibers. Several studies have documented the power of EM analysis to assess nerve regeneration (King et al., 1989; Britland et al., 1990; Llewelyn et al., 1991; Malik et al., 2001); however, this has only been applied to small numbers of patients. To apply this powerful technique to clinical trials, it is necessary to overcome two main obstacles: (1) creation of reproducible, understandable definitions for use by a large data collection team and (2) faster image capture and analyses to allow for processing of large groups of patient samples.
In the current study, a set of definitions based on the literature were developed and tested to train a group of EM readers with varying levels of experience and expertise. Images were collected using a digital camera and computer-driven stage to record the entire cross-sectional area of a given fascicle. The separate images were then combined to form a high-resolution 'virtual' image of the fascicle and presented to the readers via image analysis software. To our knowledge, this is the first application of digital technology to the ultrastructural study of a human nerve disease. We believe that this type of detailed anatomical information will provide insight into the processes of disease and recovery and will support the development of new treatment strategies and future therapeutic interventions. In addition, these techniques are applicable to any anatomically based analyses of pathology and treatment.

\section{Materials and Methods}

\section{Patients}

The patients participating in the current study were type I and type II diabetics with symptoms of neuropathy but measurable sural and peroneal responses and quantitative vibration thresholds. Sural nerve biopsies were obtained at the beginning of the treatment period and following 12 months of treatment. Patient selection criteria have been described in detail elsewhere (Sima et al., 1993a; Albers et al., 1996a; 1996b; Greene et al., 1999).

\section{Biopsy processing}

Sural nerve biopsies were collected from patients with DPN as part of a multicenter clinical study. Following surgery, the sural nerve was divided into $1-\mathrm{cm}$ segments and placed in $0.1 \mathrm{M}$ cacodylate buffer (pH 7.3) containing $2.5 \%$ glutaraldehyde. Upon arrival at the University of Michigan, the sample received a four-digit randomized identification number (RIN) to assure that the technicians and analysis team remained unaware of the treatment group. A segment designated for morphological analysis was subdivided into four segments and placed in fresh fixative for $1-2 \mathrm{~h}$ prior to automated processing including rinses, osmification, and dehydration. In brief, the sample was rinsed with $0.1 \mathrm{M}$ cacodylate buffer ( $\mathrm{pH} 7.3,2 \times, 15 \mathrm{~min})$ followed by osmification with $1 \%$ osmium $[4 \%$ sucrose, $1.5 \% \mathrm{~K}_{3} \mathrm{Fe}(\mathrm{CN})_{6}$ ] in cacodylate buffer. Following additional rinses in cacodylate buffer, the nerve sample was dehydrated through ethanol (50-100\%) followed by propylene oxide (PO), PO:Epon 812, $1: 1$, and finally Epon 812 alone. Segments were oriented cross-sectionally to the cutting surface of 
the block, embedded in Epon 812, and cured (48 h at $40^{\circ} \mathrm{C}$ ). One micrometer sections were cut on an ultramicrotome and stained with toluidine blue for initial inspection (Perkins et al., 2001).

\section{Fascicle selection and grid preparation}

A nerve fascicle is defined as a bundle of myelinated and unmyelinated fibers surrounded by a dense layer of continuous, convex connective tissue, the perineurium. The human sural nerve contains as many as 15 fascicles (Fig. 1). Prior studies have established that the MNFD of the largest fascicle is representative of whole-nerve MNFD, provided the area of the fascicle studied is larger than $100,000 \mu \mathrm{m}^{2}$ (Greene and Brown, 1995; Perkins et al., 2001). As part of the LM analysis, a fascicle selection procedure was implemented to assure the quality of a sample. Samples containing preparation artifacts were not included for analysis. Criteria for fascicle rejection include: $\geq 6 \%$ deviation from circularity and $\geq 6 \%$ of the myelinated fibers distorted by surgical trauma or incomplete fixation. To be considered for analysis, a fascicle had to be at least $100,000 \mu \mathrm{m}^{2}$ in area. For consistency, the largest, artifact-free fascicle was always selected. A fascicular area $>450,000 \mu \mathrm{m}^{2}$ or a maximum diameter of $730 \mu \mathrm{m}$ was an added criterion to ensure that the entire fascicle was visible when placed on a slot grid.

Thin sections in the $80-90 \mathrm{~nm}$ range (silver-gold interference color) were prepared using a Diatome diamond knife (Diatome US, Ft. Washington, PA) on a Reichert-Jung Ultracut E microtome (Leica Inc.,

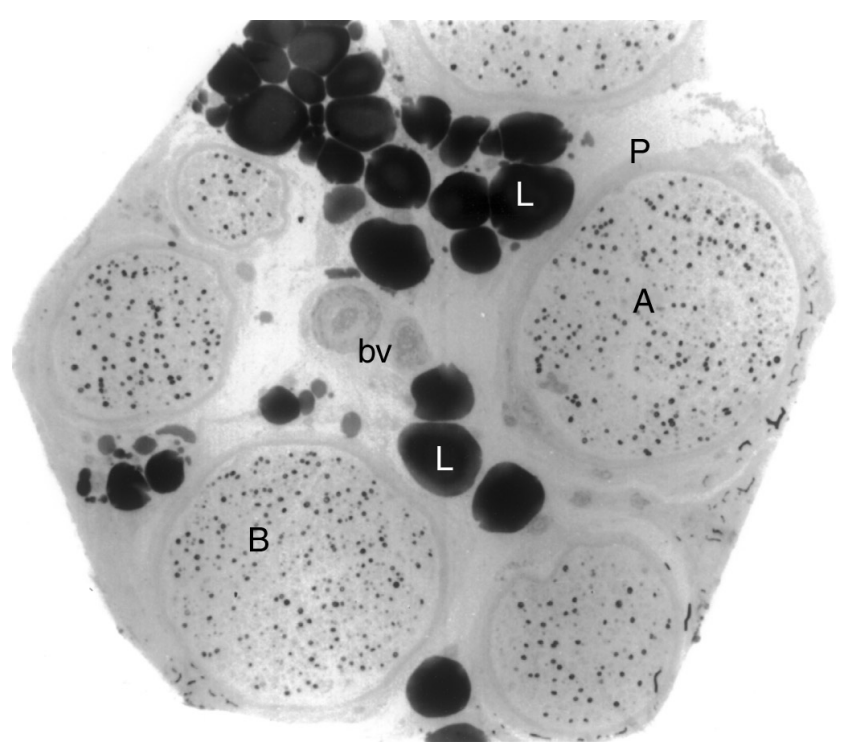

Figure1. Sural nerve biopsy. A human sural nerve contains many fascicles. In this sample, fascicles $A$ and $B$ are both free of artifact and suitable for light (LM) and electron microscopic (EM) analysis. A would be selected due to its larger size. bv, blood vessel; L, lipid; P, perineurium.
Deerfield, IL). A single section was floated onto a $2 \times 1 \mathrm{~mm}$ formvar-coated copper slot grid stabilized with evaporated carbon film (Electron Microscopy Services, Inc., Fort Washington, PA). Five grids were collected per sample. To enhance contrast, each grid was stained with uranyl acetate and lead citrate. The grids were then stabilized by sublimating spectrographically pure carbon (Ted Pella, Inc., Redding, CA) using the rotating stage of a Denton Model DV-502 vacuum evaporator (Denton, Moorestown, NJ).

\section{Digital image collection}

The stained and stabilized grid was placed in a Philips CM-100 transmission electron microscope (North American Philips Co., Mahwah, NJ) fitted with a Kodak Megaplus Camera, Model 1.6 (Eastman Kodak Co., Motion Analysis Systems Division, San Diego, CA). The movement of the grid was controlled using a computer-driven stage (CompuStage, North American Philips Co., Mahwah, NJ). The sections were exposed to the electron beam for 2 min prior to image collection to minimize section shrinkage during image capture. The digital acquisition software was initialized using the 'position' and 'radius' controls to position a bounding circle that surrounded the fascicle. The stage then stepped in a serpentine pattern with $12 \%$ vertical and $18 \%$ horizontal overlap to collect an image of the entire fascicle. The digital camera and stage movement were coordinated to capture an image every $6 \mathrm{~s}$. The average time required to collect a sample was $2-3 \mathrm{~h}$. Digital images were collected at a magnification of $\times 4600$.

The resulting individual TIF files were assembled into a 'virtual fascicle' by application of a montaging program (Vogt et al., 1996; Vogt, 1996). Specialized object recognition software identified and marked myelinated axons (Vogt et al., 1996; Vogt, 1996). Following montage construction and axon marking, the virtual image of the EM sample was presented to the EM readers on Hewlett Packard workstations using adapted Visilog software.

\section{Digital cueing procedures for EM samples and EM analysis}

These techniques enabled digital EM imaging of entire nerve fascicles at sufficient resolution to analyze the basal lamina. On average, this required 1064 individual digitally captured micrographs per fascicle. Environmental Research Institute in Michigan (ERIM) developed software to automatically register the micrographs into a single image of the entire fascicle and to automatically identify, or 'cue', the locations of myelinated fibers and clusters of myelinated fibers from the images. 
Formation of a single virtual image from large numbers of individual electron micrographs (frames) required that each micrograph be preprocessed, registered, and mosaicked. The first step in image preprocessing was to generate a background image that was divided into each frame to remove beam effects and phosphor variations. Intensity statistics were gathered from the entire set of frames and used to compute the function for contrast stretching. The contrast-stretched frames were stored as 8-bit images. The registration process determined the translation required to align adjacent images and rows of images. An initial translation was determined from the nominal stage coordinates. Subsequent steps refined the estimate, first using image correlation on sampled frames, then by a binary tree search on full resolution frames. The relative positions of horizontal rows of frames were determined from the best pairwise registration between frames near the center of the two rows. The construction of the mosaic image placed all of the registered frames into a single overall coordinate system and then divided the resulting image data into nonoverlapping $1024 \times 1024$ pixel tiles which comprised the virtual image. The high-resolution virtual image was downsampled by eight pixels in both directions to form a low-resolution image. The low-resolution image, typically $25 \mathrm{MB}$ in size, was used by the myelinated fiber cueing algorithm and for the first stage of interactive image review.

The myelinated fiber cueing algorithms were based on operators from mathematical morphology that were well suited to shape analysis in binary and gray-scale imagery. The algorithms were tailored to the review process. Because there were typically 1000-2000 myelinated fibers in each fascicle, the myelinated fiber cueing algorithm was designed to locate as many myelinated fibers as possible without generating false cues that would be time consuming to correct. Myelinated fiber cueing was performed by treating the gray-scale intensity values as a topological surface and locating regions where the characteristic tower structure of the light axon interior was surrounded by a dark moat of myelin. Boundaries were refined by recovering dark regions connected to the myelin extracted in the initial steps. The results were filtered to remove noise and linear structures, as well as fibers from adjacent fascicles.

\section{Reader training and fascicle analysis}

Development of definitions for object identification was important in setting a 'gold' standard for reader training and quality control. Standardized definitions of myelinated fibers, degenerating myelinated fibers, and regenerating myelinated fiber clusters were derived from previous studies (Sima et al., 1988; King et al., 1989; Britland et al., 1990). Previous LM studies have employed the term 'myelinated fiber' when referring to the myelinated neuronal processes within the peripheral nerve. In the current study, the term myelinated fiber refers to data described and collected at the level of the light microscope. The term 'myelinated axon' refers to data collected or described using EM. The terms and definitions follow:

\section{Regenerating myelinated axon cluster}

These are two or more myelinated axons surrounded by a common basal lamina. The continuity of the encircling basal lamina must be $90 \%$ or greater (Fig. 3A,C).

\section{Probable myelinated axon cluster}

These are two or more myelinated axons surrounded by a common basal lamina less than $90 \%$ complete (Fig. 3B).

\section{Single regenerating/remyelinating axon}

This is any single myelinated axon surrounded by an old and a new basal lamina. The basal lamina must be $90 \%$ complete (Fig. 3C).

\section{Degenerating myelinated axon}

This is a myelinated axon characterized by disruptions in or missing axoplasm. Wrinkles or folds in the myelin were not included due to possible preparation artifacts (Fig. 3D).

For readers to review these very large virtual images and record their observations, an interactive review or reading station was developed by ERIM. The reader station allowed inspection either of the low-resolution image or of selected portions of the high-resolution data. Reader training consisted of an introduction to the definitions, a review of relevant images, and instruction on the use of the analysis software. A series of test images were presented to the readers and their identification of objects compared both as a group and individually with an instructor. Over the course of the training period, consensus was achieved by comparing data sets between trainees and instructors.

A standardized sequence of procedures further assured reproducibility between readers and consistency of the reading group over time. Each reader began with a low-resolution survey of the area outside the main fascicle, the perineurium, and parts of other fascicles to remove fibers marked during the preliminary computer analysis. The reader then surveyed the fascicle for errors made by the myelinated fiber marking software and corrected these errors. Potential clusters or objects too small to identify were marked with an area of interest (AOI) mark. To make sure that all areas of the fascicle had been examined, a box enclosing a specific region was stepped across the fascicle. 
The high-resolution data could not be accessed until the reader completed the stepping function. The highresolution scan consisted of an examination of each $\mathrm{AOI}$. Following the identification of the object in question, the AOI mark was removed and the reader moved on to the next AOI. When all of the AOI marks were removed, the image reading was complete and the data set, including the myelinated axon and cluster marks, was transferred into the database.

\section{Quality control}

A subset of samples was read by two readers either internal or external to the study to address issues of reader variability. The difference between readers was not to exceed $5 \%$ for myelinated fiber counts and $10 \%$ for regenerating myelinated cluster counts. For internal quality control, a $20 \%$ subsample of the EM sections was randomly chosen and reassigned to a second internal reader. In addition, $10 \%$ of the samples were sent to another site for external quality control. A group of 24 randomly selected samples were resectioned, scanned, montaged, and read by the original reader to identify any changes in technical processing over the course of the study.

\section{Results}

\section{Fascicle selection}

The current study demonstrates the feasibility of applying digital imaging technology to the quantification of human sural nerve biopsies. As outlined in the Materials and Methods, careful examination of each biopsy ensured that only the fascicles meeting specific quality standards were examined. Application of fascicle selection criteria with regard to fixation and surgical technique resulted in samples of suitable quality for EM analysis (Fig. 1). Figure 1 is a representative example of a biopsy containing multiple fascicles of varying size. Fascicles $A$ and $B$ were acceptable by the established criteria with the final selection, fascicle A, based on fascicular area. Overall, in the 1291 biopsies received, fascicle and fiber distortions were minimal. By applying strict selection criteria (see Materials and Methods), 1153 samples were accepted for LM analysis and 1022 were accepted for EM analysis.

\section{Image collection}

The use of slot grids rather than traditional mesh grids ensured that objects of interest, in this case, myelinated axon clusters and small myelinated axons, were not obscured by the structure of the grid. Standard square mesh grids obscured $35-50 \%$ of the specimen and may lead to undersampling of rare objects and of larger objects that are likely to be partially obscured by grid bars. Digital images were collected at a magnification of $\times 4600$. Specialized software constructed a montage from the individual, overlapping digital image files (Fig. 2). Coordination of the computer-driven stage and camera ensured that no image files were omitted, and the resultant montage contained no distortions, gaps, or serious misalignments (Fig. 2). The overall result of the applied technology was a complete digital image of the fascicle of sufficient resolution to discriminate individual basal laminae necessary to identify regenerating axon clusters. This process was made more reliable by the application of carbon to stabilize the grids. Carbon stabilization of the specimen prior to exposure to the electron beam was coupled with a pre-image collection exposure to the electron beam. These procedures were necessary to maintain sample stability during the $2-3 \mathrm{~h}$ of beam time required to image the entire fascicle and were added to minimize specimen warping and stretching. Initial image collection without carbon stabilization and pre-imaging beam exposure often contained areas of specimen warping that made adjacent image correlation and alignment with simple translation impossible (data not shown). Sample stabilization techniques eliminated the need for second-order (or higher) polynomial resampling based on the tie points in the overlapping regions of adjacent images. Tie point determination and resampling to achieve image registration would have resulted in an unacceptable

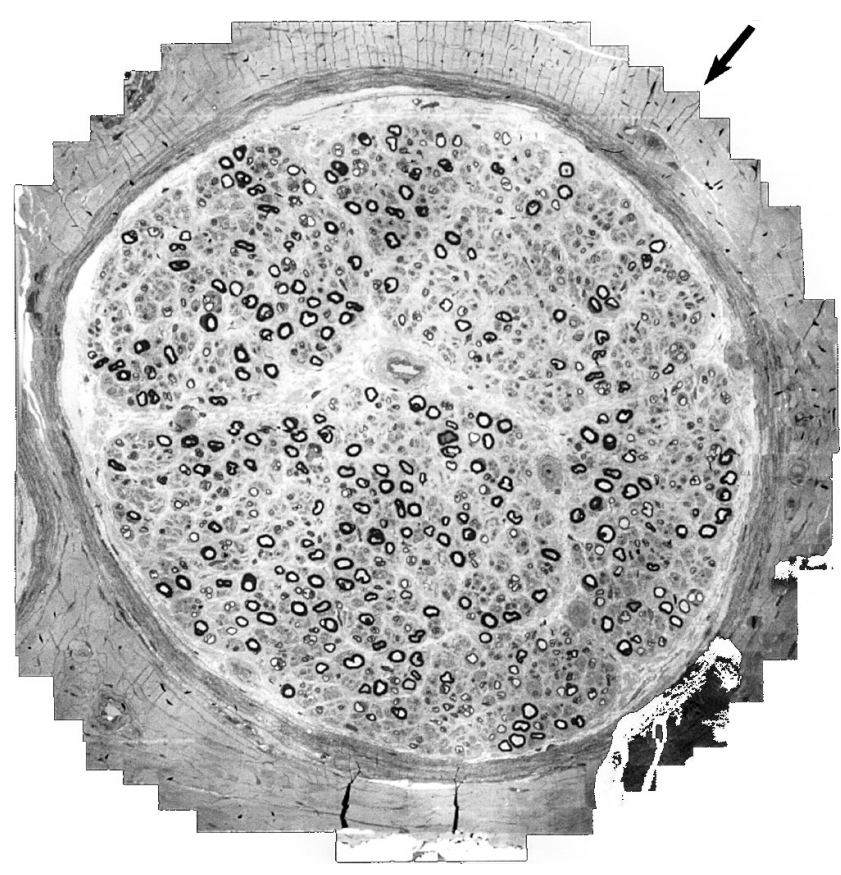

Figure 2. A completed montage of an entire sural nerve fascicle. This image is constructed of over 200 individual image tiles. Arrow indicates the edges of image tiles. 
increase in image processing time and compromised image resolution.

\section{Image analysis}

As described in Materials and Methods, a myelinated axon cueing algorithm analyzed and annotated the imagery. This was followed by myelinated axon correction and cluster identification by trained EM readers who were responsible for verifying and correcting these marks as well as identifying regenerating and degenerating myelinated axons according to the study criteria. Myelinated cluster confirmation as well as evaluation of other types of regenerative changes took place during the high-resolution scan (virtual magnification of $\times 15000$ ). The resulting data or 'marks' file appeared as an overlay of colored squares across the fascicle.

The reader interface was built using Visilog image analysis (Noesis Vision, Inc., St-Laurent, Quebec, Canada) software and was specifically tailored for its use in analyzing sural nerve biopsies. Myelinated axon (red), AOI (bright green), and degenerating axon (yellow) buttons were available for marking in the lowresolution phase of image analysis. The readers also used a three-button mouse that contained an erase feature to facilitate changes. During the high-resolution phase of analysis, a set of different colored cluster buttons were available in addition to the standard myelinated axon and degenerating axon buttons. These colors were instituted to aid in marking separate regenerating myelinated axon clusters. To annotate the imagery, the reader would click on the type of myelinated axon (normal, degenerating, or regenerating) on the side panel, then return to the imagery, and place the mark over the center of the axon. As the fascicle was reviewed, marks could be changed; however, once the reader completed the fascicle, the data were sent to the database and could no longer be altered.

\section{Normal, regenerating and degenerating axon analysis}

In 407 of 486 cases, the fascicle analyzed by EM was the same as that analyzed by LM. In 79 samples, the second largest fascicle (total area within $10 \%$ of the area of the largest) was chosen for EM due to the selection criteria (see Materials and Methods). When the same fascicle was analyzed for both LM and EM, it was possible to compare the total myelinated fiber, using LM, and myelinated axon, using EM, counts determined by these techniques. The average number of myelinated fibers identified by LM was $766 \pm 21$ (mean \pm standard error) with a range of 51-2899. The average number of total myelinated axons identified by EM was $770 \pm 21$ with a range of 47-2894. The mean difference between the number of myelinated fibers and the number of myelinated axons was $3.8 \pm 0.8$ per fascicle (mean $\pm S E$ ), $p<0.0001$. Ninety percent of the differences were no greater than 24 myelinated fibers or axons (or $3 \%$ of the average number). As expected, the average number of myelinated axons (EM) identified was slightly higher than myelinated fibers (LM); however, the difference was only $0.5 \%$ of the average.

Potential regenerating myelinated axon clusters typically appeared as small, tightly grouped myelinated fibers in the low-resolution scan. When viewed at high resolution $(\times 15000)$, the continuity of the basal lamina determined cluster classification as definite regenerating (Fig. 3A,C) or probable regenerating (Fig. 3B). In the absence of basal lamina, myelinated axons were counted as normal, single axons. Figure 3 clearly demonstrates both the quality and the resolution of the images analyzed in this study. The basal laminae appear as distinct structures encircling groups of myelinated axons (regenerating clusters) or as empty loops and fragments within the fascicle, distinct from the surrounding collagen, SC debris, and fibroblast extensions (Fig. 3A-D).

Other types of regenerative changes included single myelinated axons surrounded by multiple layers of basal lamina and myelinated axon clusters containing only one myelinated but several unmyelinated axons (Fig. 3C). Single myelinated axons with more than one basal lamina layer may be due to demyelination followed by remyelination; however, they lacked the layers of SCs and SC debris typical of onion bulb formation. When detected, these myelinated axons were labeled single clusters. Axon clusters containing a single myelinated and several unmyelinated axons were given no special designation, and only the myelinated axon was counted. Both of these myelinated axon categories appeared as normal, single myelinated axons in the low-resolution scan and were discovered only incidentally while viewing the imagery at high resolution. Therefore, while these fibers were noted in the database, their frequency could not be quantified. The end stages of degenerating myelinated axons, characterized by loss of axoplasm and presence of myelin debris were visible at low resolution as dark axons and were confirmed and quantified at high resolution (Fig. 3D).

\section{Quality control}

To assess reader variability over the course of the study, 87 samples were re-assigned and re-read for internal quality control. Myelinated axon counts did not vary by more than $5 \%$ between the original and quality control reads. For regenerating myelinated cluster counts, 23 samples varied between readers by $\geq 4$ regenerating fiber clusters. Of these differences, 

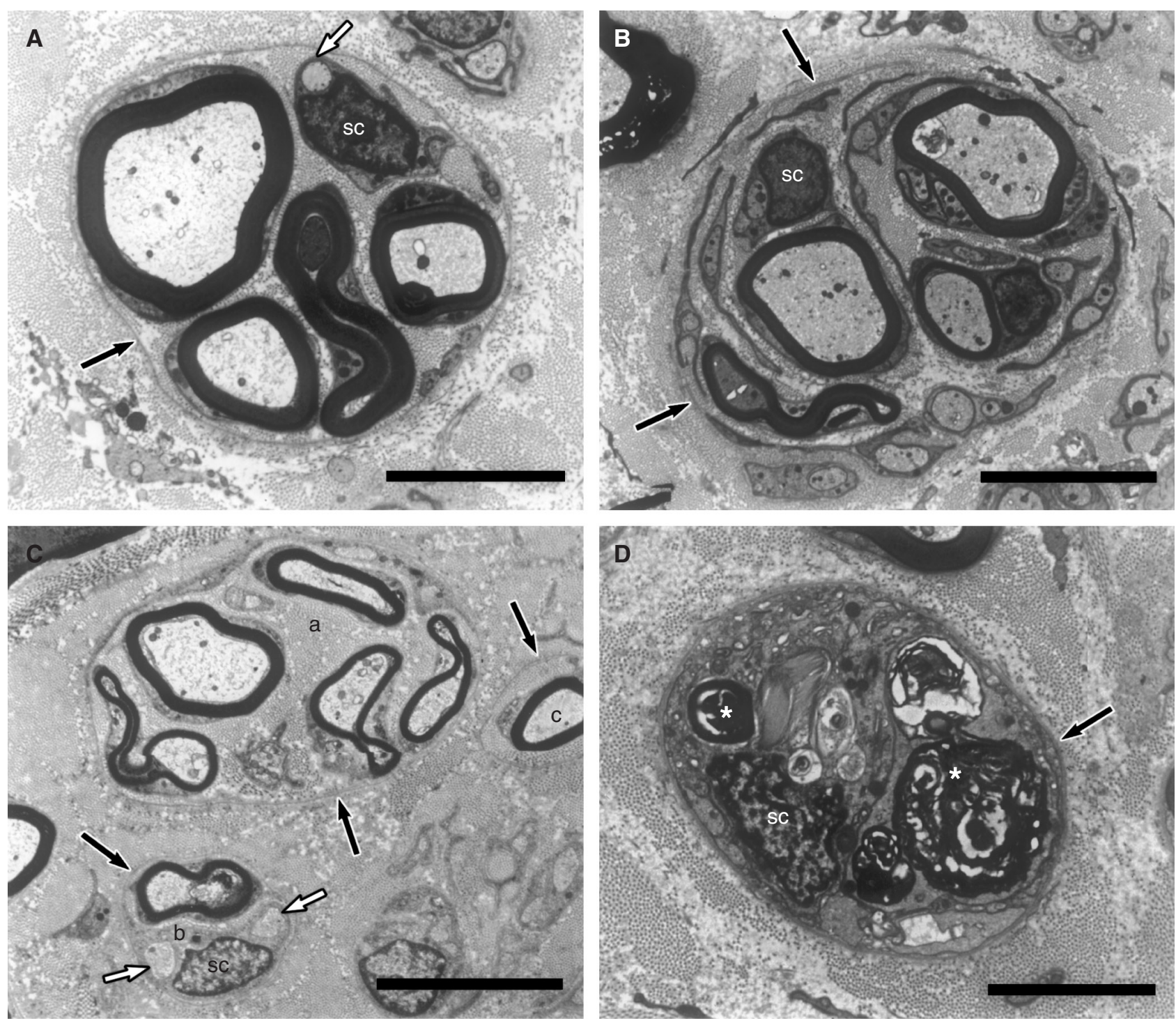

Figure 3. (A) An example of a regenerating myelinated axon cluster containing four myelinated axons in a common basal lamina (black arrow). Note the presence of an axon in the earliest stages of myelination (white arrow). (B) An example of a probable myelinated axon cluster in which the axons are tightly grouped but the basal lamina (black arrows) is less than $90 \%$ complete. (C) In addition to standard myelinated axon clusters (a), other types of regeneration are observed including myelinated and unmyelinated axons (white arrows) within a common basal lamina (b) and single myelinated axons surrounded by more that one basal lamina (c, black arrow). (D) Degenerating myelinated axons are characterized by loss of axoplasm and subsequent myelin breakdown. No axoplasm is detected in this degenerating axon and myelin debris is prominent (asterisk). Black arrows, basal lamina; SC, Schwann cell nucleus. Bar $=5 \mu \mathrm{m}$.

seven samples were severely discrepant between the original and quality control read. Thirty-six samples were sent to another laboratory for external quality control. Of these, six samples differed by more than 10 regenerating clusters. Twenty-four samples were re-sectioned, re-imaged, re-masked, and re-analyzed to assess any changes in sectioning or image processing that may have occurred over the course of the study. Of these 24 , only four samples varied by more than four regenerating fiber clusters.
The average density of definite regenerating myelinated axon clusters per $\mathrm{mm}^{2}$ was $65.8 \pm 5.1$ with a range of $0-412(n=193)$. About $8.5 \%$ of samples contained no regenerating myelinated axon clusters. The minimum number of myelinated axons with a regenerating cluster was two; however, clusters contained up to nine myelinated axons, with an average of $2.23 \pm$ myelinated axons per regenerating cluster. An average of 10.8 clusters per fascicle contained two myelinated axons, 2.2 contained three myelinated axons, and only 
0.53 clusters per fascicle contained four or more myelinated axons.

\section{Discussion}

Early in the course of diabetes, alterations in nerve metabolism result in nerve ischemia, myelinated fiber loss and the signs and symptoms of DPN (Stevens et al., 1996; Greene et al., 1997; Feldman et al., 1997; Sundkvist et al., 2000). Treatment of patients with DPN is aimed at preventing fiber loss and reducing or at least stabilizing neurological deficits. Experimental treatment for DPN in clinical trials includes compounds that inhibit the aldose reductase pathway (Greene et al., 1993; 1999; Sima et al., 1993b; Stevens et al., 1994; Hotta, 1995), restore essential fatty acids (Zenobi et al., 1993; Stevens et al., 1993a; Driscoll et al., 1994; Nickander et al., 1996; Garrett et al., 1997), reduce oxidative stress (Hellweg and Hartung, 1990; Low and Nickander, 1991; Wolf, 1993; Cameron and Cotter, 1995; Love et al., 1996), or increase levels of neurotrophic molecules (Diemel et al., 1994; Pfeiffer and Schatz, 1995; Whitworth et al., 1995). Pre- and posttreatment assessment of neuropathy was based on electrophysiology and sensory testing as primary endpoints of nerve function (Feldman et al., 1994a; 1994b; Greene et al., 1997; 1999). Morphologic analysis of myelinated nerve fibers included teased fiber analysis and counts of myelinated fibers at the level of the light microscope (Ochoa and Mair, 1969; Said et al., 1983; Jacobs and Love, 1985; Dyck et al., 1986; Llewelyn et al., 1988; Sima et al., 1988) as secondary endpoints. Measures of glucose metabolites and polyols were also assessed in trials of aldose reductase inhibitors (Greene et al., 1998).

Decreases in MNFD and increased abnormal fibers (teased fiber analysis) have been extensively reported for DPN (Greene et al., 1997) and are highly correlated with decreased nerve function and sensory loss (Greene et al., 1997). In addition, many reports in the literature describe in detail the ultrastructural changes that accompany DPN (King et al., 1989; Britland et al., 1990; Llewelyn et al., 1991; Malik et al., 2001). These studies were performed by intensive examination of small numbers of patient samples. While myelinated fiber analysis yields valuable information concerning overall MNFD, it cannot make clear distinctions between normal fibers, degenerating fibers, and small regenerating fibers. This inability to distinguish small, regenerating myelinated axons from those spared by treatment may lead to discrepancies in estimations of myelinated axons undergoing regeneration. Therefore, our goal was to find a way to combine the power of EM, the time-saving aspects of digital technology, and our experience with standardization for clinical trials. In the current study, we established a method for the digital collection, analysis and storage of large images, and data sets necessary for use in clinical trials and their automated presentation to multiple readers. To our knowledge, this is the first clinical trial to examine this number of human biopsies at this level of detail.

Our reported myelinated cluster density of $65.8 \pm 5.1 / \mathrm{mm}^{2}$ is substantially lower than that reported by Britland et al. (1990) and Llewelyn et al. (1991). These differences may be because of multiple factors including the use of conservative definitions of regeneration and area sampled. As mentioned above, the goals of the current study were to optimize protocols by which large numbers of samples could be analyzed by multiple readers. To this end, a regenerating myelinated axon cluster was defined as two or more myelinated axons surrounded by a common basal lamina that was $90 \%$ complete. Definitions of regeneration in prior studies include Büngner Bands containing only unmyelinated axons, clusters containing as few as one myelinated and several unmyelinated axons (Britland et al., 1990), or two or more myelinated axons in close apposition with or without an encircling basal lamina (Llewelyn et al., 1991). It is clear from our observations and those of others (King et al., 1989; Britland et al., 1990; Llewelyn et al., 1991) that total regenerative changes span a broad scope. The inclusion of 'probable' clusters in the database was an attempt to track other types of regeneration. Consensus concerning basal lamina integrity below $90 \%$ was not possible to achieve in the study time frame. Therefore, the probable classification was added to re-address these clusters if further analysis was warranted. While the definitions used in the current study characterize only one stage of regeneration, the data are available for further in depth analysis based on the results of treatment, positive or negative, or other signs and symptoms reported by patients or clinicians involved in the study. Thus, our data provide a deeper examination of the anatomical effects of disease and treatment with the added benefit that further analysis does not require resampling of the patients or reprocessing of samples.

We believed that because regenerating clusters would be rare and their distribution across a given fascicle was unknown, it was important to sample as large an area as possible. Analysis of the largest fascicle was chosen to take advantage of the known myelinated fiber densities gathered by LM. This measurement paradigm has been implemented in other drug trials (Perkins et al., 2001) with statistically significant results. While previous studies described in detail the type of regenerative changes examined, little detail was included concerning the sampling 
procedure. Sampling paradigms for LM include analysis of the largest fascicle, random frame grabbing, and counting all possible frames (Tomlinson and Mayer, 1984; Britland et al., 1990; Llewelyn et al., 1991). Digital EM could accommodate many of these sampling paradigms. Multiple fascicles may fit onto $1.5 \times 2 \mathrm{~mm}$ slot or $2.0 \mathrm{~mm}$ hole grids. In this way, several fascicles could be imaged or random frames collected by setting the stage to sample at a given spatial interval.

Previous EM examinations of nerve biopsies have been limited by the use of copper mesh grids (Sima et al., 1988; Britland et al., 1990; Greene et al., 1997), photographic imaging (Sima et al., 1988; Britland et al., 1990; Greene et al., 1997), and the time needed for these processes. Up to $50 \%$ of the imagery is obscured by copper mesh grids. Traditional photographic methods of image capture on EM film would result in 150-200 negatives to develop and subsequently print as $8 \times 10$ photomicrographs. These photomicrographs would then have to be physically mosaicked and analyzed by visual inspection and manual data compilation. The resulting photographic montage would be approximately 30 feet in diameter. The overall time required for analysis at the EM level is somewhat longer than the time for analysis at the light level. In general, tissue processing is the same for both types of analyses, as adequate resolution is only achieved by plastic sections. LM analysis is performed semi-automatically by scanning and analyzing at the same time, frame by frame. The advantage is that image capture and analysis are concurrent and the disadvantage is that the work station must be constantly attended and the program uninterrupted from start to finish. This procedure can take anywhere from 2 to $8 \mathrm{~h}$ to complete, depending on the size of the fascicle measured.

In EM analysis, image capture, processing, and automated analysis can take from 3 to $8 \mathrm{~h}$ followed by interactive analysis which can take from 2 to $5 \mathrm{~h}$. The added time may be prohibitive for some studies; however, the depth of analysis made possible by capturing high-resolution ultrastructure cannot be overestimated and additional LM analysis would not be required. The current study used simple definitions to define regenerative clusters to assure consistency and to expedite the analyses. A deeper analysis of the data may be performed on a subset of entire fascicles or on a limited number of frames from all the fascicles in the study. This work could be performed by more highly trained personnel and form the basis for future definitions and study endpoints. For example, regenerating clusters may be further defined by the number of sprouts within a cluster, the total myelinated axon size and myelin thickness, and the completeness of the basal lamina. These characteristics may be used to determine the difference between newly formed regenerative clusters (complete basal lamina and higher number of fiber sprouts) and those that are more mature (basal lamina breaking down and fewer fiber sprouts) (King et al., 1989; Britland et al., 1990; Llewelyn et al., 1991; Sundkvist et al., 2000). Additionally, algorithms could be developed to relate the location of regenerating clusters to other structures including degenerating myelinated axons and blood vessels as well as distance from the perineurium and each other. Taken together, these data allow for comparisons of rates of degeneration to regeneration in response to treatment. When applied to animal studies, these techniques and analyses may help model diffusion and availability of drugs or growth factors in future preclinical pharmaceutical trials. In addition to regenerative cluster analysis, axon size and myelin thickness could be assessed by applying new algorithms to the existing data set. In this way, demyelination could also be assessed.

The EM readers for the study included three Ph.D. level anatomists and an EM technician with 10 years of experience. This group easily identified axons in various stages of degeneration and regeneration at high resolution. However, the features of these axons could not be assessed during the initial low-resolution scan. This was also problematic for some regenerative axon clusters. The readers learned to err on the side of caution and place AOI marks on any group of axons that even remotely appeared as though it may be an axon cluster. This point emphasizes the need for a multi-tiered analysis strategy. This could easily be implemented by extending the training of the initial analysis team or by sending selected images to experts in the field of neuropathology.

Quality control was maintained over the course of the study by the application of standardized reader training and reading procedures. This included a 6-8week reader training period during which the readers were familiarized with the imagery to be analyzed as well as the software to be used. Readers were not allowed to collect data until certified by the project statistician. This process involved comparison of the reader's data set to an instructor data set collected from a specific set of images. Requirements for certification include agreement within 5\% for total fiber counts and agreement within $10 \%$ for total regenerating cluster counts.

Recent advances in existing image analysis software including MetaMorph (Universal Imaging, Downington, PA) and Image-Pro (Media Cybernetics, Silver Spring, MD) may make this process available to a wider group of scientists. When applied to clinical trials, great care must be taken to ensure consistency in all aspects of data collection from processing and 
preparation of samples, instrumentation, and reader training. For both large and small studies, automated image analysis greatly reduces fatigue and provides a direct link between data collection and storage, as the data can be analyzed by the image analysis software or directly exported to Excel or a similar data handling software. These techniques apply to any disease model in which biopsies are routinely analyzed.

The above described methods and results indicate that digital analyses of human sural nerve biopsies are possible and feasible. The degree of analysis may be extended beyond the current work by examining the entire nerve rather than the largest fascicle and/or by analyzing selected frames in more detail than outlined here. In summary, the use of digital EM, a powerful computer-assisted image analysis and multiple readers for data collection, enabled the ultrastructural examination of important regenerative changes in a multi-center clinical trial. Future examinations of this and other archived biopsy material could include investigations of all types of regenerative changes as well as an examination of the number and condition of the unmyelinated fibers. Study of placebo-treated patients in addition to experimentally treated patients will greatly contribute to our understanding of the progression of DPN and the ultrastructural underpinnings of drug therapies.

\section{Acknowledgements}

The authors thank Ms. J.C. Boldt for expert secretarial assistance and Mr. J.L. Beals for expert digital image construction and annotation. The authors also acknowledge the insightful discussions of the manuscript by Dr. E.L. Feldman. Mr. C. Edwards of the Cell Biology Laboratory, University of Michigan provided electron microscopy support. This study was made possible by the cooperation between the Division of Endocrinology and Metabolism, Michigan Diabetes and Research Training Center, Juvenile Diabetes Research Foundation International, the Department of Cell and Developmental Biology of the University of Michigan and ERIM International. This work was conducted with the support of Hoffmann-LaRoche, Canada.

\section{References}

Albers JW, Brown MB, Sima AAF, Greene DA (1996a). Frequency of median mononeuropathy in patients with mild diabetic neuropathy in the early diabetes intervention trial (EDIT). Muscle Nerve 19:140-146.

Albers JW, Brown MB, Sima AAF, Greene DA (1996b). Nerve conduction measures in mild diabetic neuropathy in the Early Diabetes Intervention Trial: The effects of age, sex, type of diabetes, disease duration, and anthropometric factors. Neurology 46:85-91.
Black MM, Lasek RJ (1980). Slow components of axonal transport: two cytoskeletal networks. J Cell Biol 86:616-623.

Britland ST, Young RJ, Sharma AK, Clarke BF (1990). Association of painful and painless diabetic polyneuropathy with different patterns of nerve fiber degeneration and regeneration. Diabetes 39:898-908.

Cameron NE, Cotter MA (1995). Neurovascular dysfunction in diabetic rats. Potential contribution of autoxidation and free radicals examined using transition metal chelating agents. J Clin Invest 96:1159-1163.

Comi G, Fedele D, Coscelli C, Cucinotta D, Feldman EL, Ghirlanda G, Negrin P, Santeusanio F, Maderna L, Italian Diabetic Neuropathy Committee (1999). The Italian multicentre study on the prevalence of distal symmetric polyneuropathy: correlation between clinical variables and nerve conduction parameters. In: Clinical Neurophysiology: From Receptors to Perception (EEG Suppl. 50). Comi G, Lucking $\mathrm{CH}$, Kimura J, Rossini PM (Eds). Elsevier Science, Cambridge, UK, pp 546-552.

Diemel LT, Brewster WJ, Fernyhough P, Tomlinson DR (1994). Expression of neuropeptides in experimental diabetes; effects of treatment with nerve growth factor or brainderived neurotrophic factor. Brain Res Mol Brain Res 21: 171-175.

Driscoll D, Ennis W, Meneses P (1994). Human sciatic nerve phospholipid profiles from non-diabetes mellitus, non-insulindependent diabetes mellitus and insulin-dependent diabetes mellitus individuals. A ${ }^{31} \mathrm{P}$ NMR spectroscopy study. Int J Biochem 26:759-767.

Dyck PJ, Lais A, Karnes JL, O’Brien P, Rizza R (1986). Fiber loss is primary and multifocal in sural nerves in diabetic polyneuropathy. Ann Neurol 19:425-439.

Feldman EL, Stevens MJ, Greene DA (1994a). Treatment of diabetic neuropathy. In: Advances in Endocrinology and Metabolism. Mazzaferri EL, Bar RS, Kreisberg RA (Eds). Mosby Year Book, St Louis, MO, pp 393-428.

Feldman EL, Stevens MJ, Greene DA (1997). Pathogenesis of diabetic neuropathy. Clin Neurosci 4:365-370.

Feldman EL, Stevens MJ, Thomas PK, Brown MB, Canal N, Greene DA (1994b). A practical two-step quantitative clinical and electrophysiological assessment for the diagnosis and staging of diabetic neuropathy. Diabetes Care 17: 1281-1289.

Garrett NE, Malcangio M, Dewhurst M, Tomlinson DR (1997). $\alpha$-lipoic acid corrects neuropeptide deficits in diabetic rats via induction of trophic support. Neurosci Lett 222:191-194.

Greene DA, Arezzo JC, Brown MB (1999). Effect of aldose reductase inhibition on nerve conduction and morphometry in diabetic neuropathy. Zenarestat Study Group. Neurology 53:580-591.

Greene DA, Brown MB (1995). Validation of sural nerve fiber density and percent normal teased fibers as morphological endpoints in clinical trials of diabetic neuropathy. In: Diabetic Neuropathy: New Concepts and Insights. Hotta N, Greene DA, Ward JD, Sima AAF, Boulton AJM (Eds). Exerpta Medica, Elsevier, Cambridge, UK, pp 379-385.

Greene DA, Feldman EL, Stevens MJ, Sima AAF, Albers JW, Pfeifer MA (1997). Diabetic neuropathy. In: Diabetes Mellitus. Porte D Jr, Sherwin R (Eds). Appleton \& Lange, Norwalk, CT, pp 1009-1076.

Greene DA, Sima AAF, Stevens MJ, Feldman EL, Killen PD, Henry DN, Thomas T, Dananberg J, Lattimer SA (1993). 
Aldose reductase inhibitors: An approach to the treatment of diabetic nerve damage. Diabetes Metab Rev 9:189-217.

Hellweg R, Hartung H-D (1990). Endogenous levels of nerve growth factor (NGF) are altered in experimental diabetes mellitus: a possible role for NGF in the pathogenesis of diabetic neuropathy. J Neurosci Res 26:258-267.

Herman WH, Sinnock P, Brenner E, Brimberry JL, Langford D, Nakashima A, Sepe SJ, Teutsch SM, Mazze RS (1984). An epidemiologic model for diabetes mellitus: incidence, prevalence, and mortality. Diabetes Care 7:367-371.

Hotta N (1995). New approaches for treatment in diabetes: Aldose reductase inhibitors. Biomed Pharmacother 49:232-243.

Jacobs JM, Love S (1985). Qualitative and quantitative morphology of human sural nerve at different ages. Brain 108:897-924.

King RHM, Llewelyn JG, Thomas PK, Gilbey SG, Watkins PJ (1989). Diabetic neuropathy: Abnormalities of Schwann cell and perineurial basal laminae. Implications for diabetic vasculopathy. Neuropathol Appl Neurobiol 15:339-355.

Llewelyn JG, Gilbey SG, Thomas PK, King RHM, Muddle JR, Watkins PJ (1991). Sural nerve morphometry in diabetic autonomic and painful sensory neuropathy. Brain 114:867-892.

Llewelyn JG, Thomas PK, Gilbey SG, Watkins PJ, Muddle JR (1988). Pattern of myelinated fibre loss in the sural nerve in neuropathy related to type 1 (insulin-dependent) diabetes. Diabetologia 31:162-167.

Love A, Cotter MA, Cameron NE (1996). Nerve function and regeneration in diabetic and galactosaemic rats: Antioxidant and metal chelator effects. Eur J Pharmacol 314:33-39.

Low PA, Nickander KK (1991). Oxygen free radical effects in sciatic nerve in experimental diabetes. Diabetes 40:873-877.

Malik RA, Veves A, Walker D, Siddique I, Lye RH, Schady W, Boulton AJ (2001). Sural nerve fibre pathology in diabetic patients with mild neuropathy: relationship to pain, quantitative sensory testing and peripheral nerve electrophysiology. Acta Neuropathol 101:367-374.

Nickander KK, McPhee BR, Low PA, Tritschler H (1996). $\alpha$-lipoic acid: Antioxidant potency against lipid peroxidation of neural tissues in vitro and implications for diabetic neuropathy. Free Radic Biol Med 21:631-639.

Ochoa J, Mair WGP (1969). The normal sural nerve in man: ultrastructure and numbers of fibres and cells. Acta Neuropathol 13:197-216.

Perkins BA, Greene DA, Bril V (2001). Glycemic control is related to the morphological severity of diabetic sensorimotor polyneuropathy. Diabetes Care 24:748-752.

Pfeiffer A, Schatz H (1995). Diabetic microvascular complications and growth factors. Exp Clin Endocrinol 103:7-14.

Said G (1996). Diabetic neuropathy: an update. J Neurol 243: 431-440.

Said G, Slama G, Selva J (1983). Progressive centripetal degeneration of axons in small fibre diabetic polyneuropathy. Brain 106:791-807.

Sima AAF, Bril V, Nathaniel V, McEwen TAJ, Brown MB, Lattimer SA, Greene DA (1988). Regeneration and repair of myelinated fibers in sural-nerve biopsy specimens from patients with diabetic neuropathy treated with sorbinil. N Engl J Med 319:548-555.
Sima AA, Greene DA, Brown MB, Hohman TC, Hicks D, Graepel GJ, Bochenek WJ, Beg M, Gonen B (1993a). Effect of hyperglycemia and the aldose reductase inhibitor tolrestat on sural nerve biochemistry and morphometry in advanced diabetic peripheral polyneuropathy. The Tolrestat Study Group. J Diabetes Complications 7:157-169.

Sima AAF, Stevens MJ, Feldman EL, Cherian PV, Greene DA (1993b). Animal models as tools for the testing of preventive and therapeutic measures in diabetic neuropathy. In: Lessons from Animal Diabetes IV. Shafrir E (Ed). Smith-Gordon, London, pp 177-191.

Stevens MJ, Dananberg J, Feldman EL, Lattimer SA, Kamijo M, Thomas TP, Shindo H, Sima AAF, Greene DA (1994). The linked roles of nitric oxide, aldose reductase $\left(\mathrm{Na}^{+}, \mathrm{K}^{+}\right)$-ATPase in the slowing of nerve conduction in the streptozotocin diabetic rat. J Clin Invest 94:853-859.

Stevens MJ, Feldman EL, Funnell MM, Sima AAF, Greene DA (1993b). Optimal methods for detecting early neuropathy and its progression. In: Diabetes Forum IV Concepts for the Ideal Diabetes Clinic. Mogensen CE, Standl E (Eds). pp 315-332.

Stevens MJ, Lattimer SA, Feldman EL, Helton ED, Millington DS, Sima AAF, Greene DA (1996). Acetyl-L-carnitine deficiency as a cause of altered nerve myo-inositol content, $\mathrm{Na}$, K-ATPase activity and motor conduction velocity in the streptozotocin-diabetic rat. Metabolism 45:865-872.

Stevens EJ, Lockett MJ, Carrington AL, Tomlinson DR (1993a). Essential fatty acid treatment prevents nerve ischaemia and associated conduction anomalies in rats with experimental diabetes mellitus. Diabetologia 36:397-401.

Sundkvist G, Dahlin LB, Nilsson $H$, Eriksson KF, Lindgarde $F$, Rosen I, Lattimer SA, Sima AAF, Sullivan K, Greene DA (2000). Sorbitol and myo-inositol levels and morphology of sural nerve in relation to peripheral nerve function and clinical neuropathy in men with diabetic, impaired, and normal glucose tolerance. Diabet Med 17:259-268.

Tomlinson DR, Mayer JH (1984). Defects of axonal transport in diabetes mellitus-a possible contribution to the aetiology of diabetic neuropathy. J Auton Pharmacol 4:59-72.

Vogt RC (1996). Robust extraction of axon fibers from large-scale electron micrograph mosaics. International Symposium on Mathematical Morphology.

Vogt RC, Trenkle JM, Harmon LA (1996). Mosaic construction, processing and review of very large electron micrograph composites. International Symposium on Optical Science, Engineering, and Instrumentation.

Whitworth IH, Terenghi G, Green CJ, Brown RA, Stevens E, Tomlinson DR (1995). Targeted delivery of nerve growth factor via fibronectin conduits assists nerve regeneration in control and diabetic rats. Eur J Neurosci 7:2220-2225.

Wolf SP (1993). Transition metals and oxidative stress in the complications of diabetes. In: The Role of Anti-Oxidants in Diabetes Mellitus. Gries FA, Wessels K (Eds). pmi-Verlagsgrupe, London, pp 82-101.

Zenobi PD, Holzmann P, Glatz Y, Riesen WF, Froesch ER (1993). Improvement of lipid profile in Type 2 (non-insulin-dependent) diabetes mellitus by insulin-like growth factor I. Diabetologia 36:465-469. 